\title{
Comparison of Time Lapse Embryo Morphokinetics Monitoring and Conventional Pronuclear Morphology Assessment to Determine Embryo Quality in Endometriosis Patients
}

Corresponding Author: R. Muharam; email: rmuharam@yahoo.com

Received: 24 August 2016 Accepted: 25 September 2016 Published: 4 October 2016

Publishing services provided by Knowledge E

(c) R. Muharam et al. This article is distributed under the terms of the Creative

commons Attribution

License, which permits unrestricted use and redistribution provided that the original author and source are credited.

Selection and Peer-review under the responsibility of the ASPIRE Conference Committee.

\section{R. Muharam ${ }^{1,2}$, Muhammad Ikhsan², Wisnu Setiyawan ${ }^{3}$, Malvin Emeraldi ${ }^{3}$, and Budi Wiweko}

${ }^{1} Y a s m i n$ IVF Clinic, Cipto Mangunkusumo General Hospital, Jakarta, Indonesia ${ }^{2}$ Indonesian Reproductive Medicine Research and Training Center, Faculty of Medicine Universitas Indonesia, Jakarta, Indonesia

${ }^{3}$ Department of Obstetrics and Gynecology, Faculty of Medicine Universitas Indonesia, Jakarta, Indonesia

\section{Abstract}

Objective. Endometriosis patients who underwent IVF program has poorer embryo quality, leads to low successive rate. In order to determine embryo quality, time lapse embryo monitoring and pronuclear (PN) morphology assessment were used to predict and select the most promising embryo. This research is expected to exhibit comparison the use of both methods in endometriosis patients in order to implement the most effective evaluation in future embryo implantation prognosis. Methods. This cohort research was conducted to 72 embryos from 19 endometriosis patients who underwent IVF program in Yasmin Reproductive Clinic, Cipto Mangunkusumo Hospital. After ICSI was performed two-nucleated embryo later be monitored by its cleavage time and would be classified into categories for good quality embryo and other classes were considered poor quality embryo for time lapse monitoring. Then from time lapse monitoring, PN morphology were assessed regarding pronuclear scoring. Results. Based on 72 embryos observation, we found that there was no significant difference between good and poor quality embryo based on PN scoring with $p=0.185$, and also between time-lapse morphokinetic monitoring and embryo quality with $p=0.526$. However, we found that there was a weak correlation between PN scoring and embryo quality with $p=0.049$ and $r=0.233$. Conclusion. There was no significant difference found between time lapse monitoring and PN scoring in endometriosis patients in order to determine embryo quality. However, there was a weak correlation between PN scoring and embryo quality. This might be due to small number of samples taken and visual bias during observation.

Keywords: time-lapse, pronuclear, embryo quality, endometriosis

\section{G OPEN ACCESS}




\section{Introduction}

About $50 \%$ of endometriosis patients have subfertility problem. The exact mechanism between endometriosis and infertility are still not clear. And it's still a controversy whether endometriosis is really affecting fertility or just a coincidental finding [1].

Endometriosis is a condition which the appearance of endometrial-like tissue (whether stroma or glandular tissue) which abnormally implanted outside uterine cavity. Endometriotic tissue is able to response with hormonal cycle of normal menstrual cycle. Microscopic internal bleeding along with inflammatory response, neovascularization, and scar tissue formation contribute to clinical manifestations of endometriosis patients [2]. Typical symptoms of endometriosis vary between menstrual pain (dysmenorrheal), dyspareunia, dyskezia, chronic pelvic pain, and infertility [3].

The mechanisms of infertility in endometriosis patients may vary between folicullogenesis, ovulatory, and fertility disturbance, embryo development, and even with the implantation of embryos [4]. To deal with infertility problem, endometriosis patients may choose two of the following intervention, surgical intervention or assisted reproductive technology (ART). In moderate to severe cases of endometriosis, in vitro fertilization (IFV) may improve fecundity per cycle and give a better hope to deal with infertility. However, a meta-analysis of observational studies showed that endometriosis patients with infertility had lower chance of implantation and clinical pregnancy compared to other conditions in IVF program [5].

Low successful rate of IVF program in endometriosis patients might be caused of several factors including low oocytes quality and number, reduced number of embryos attained, and poorer embryos quality [6]. To monitor embryos quality and development, which contributing to success of IVF program, several methods could be used. Those methods are static morphological assessment, preimplantation genetic diagnostic, transcryptomic, metabolomic and proteinomic, and simultaneous assessment with microscope (time-lapse monitoring) [7].

Static morphological assessment such as pronuclear morphology assessment has benefits of easier and cheaper to do. However, this method have some disadvantages including limited embryos developmental monitoring and embryos displacement during assessment itself might bring physical stress to the embryos [7, 8]. Assessment with time-lapse monitoring allows us to monitor embryos morphologies simultaneously. Time-lapse monitoring assessment may identify early morphological marker thus predicting of good quality embryos which affecting to success of embryos implantation [9].

In this study, we compared conventional pronuclear morphology assessment and morphokinetic assessment using time-lapse monitoring to predict embryos quality in endometriosis patients. This study also evaluated whether conventional static morphological assessment still could be used to predict embryos quality, considering that time-lapse monitoring tools are still limited in Indonesia. 


\section{Materials and Methods}

This is an observational study with prospective cohort approach to assess conventional pronuclear morphology and morphokinetic using time-lapse monitoring to predict embryos quality in endometriosis patients. Data was taken in sequential images of time-lapse.

The study conducted in Yasmin IVF Clinic, Cipto Mangunkusumo National General Hospital, Jakarta, Indonesia between January 2015 and January 2016. Target population of this study was all patients with endometriosis who underwent IVF procedure in Indonesia. Samples were taken from all endometriosis patients who underwent IVF procedure in Yasmin Clinic, Cipto Mangunkusumo National Hospital. Samples collected with consecutive sampling methods that fulfilled inclusions criteria and failed exclusions criteria.

The inclusions criteria of this study consist of subjects willing to participate, patients with unilateral or bilateral endometriosis who underwent surgery (proved by histopathology of endometriosis). Exclusions criteria in this study were no mature oocyte retrieved, delayed IVF procedure, and abnormality on sperm analysis.

Primary data was taken directly from every subject in the study. Every subject had been informed about backgrounds, goals, risks and benefits of the study. Subjects who agreed to be enrolled in the study were asked to sign the consent. This study has been approved by ethic committee Medical Faculty University of Indonesia, Jakarta, Indonesia.

Sample collection started with oocytes retrieval, embryos culture, and time-lapse monitoring. We used appropriate medium for every stage of embryo development (medium for gamete, fertilization, cleavage, and blastocytes (Origio, Denmark)). After ICSI (intracytoplasmic sperm injection) only embryos with prominent pronuclear included for the next step. Embryos attained were placed into separated wells designated inside the specified culture dish with digital inversion microscope (Primovision dish, Cryo-Innovation Ltd., Hungary). Then, the embryos would be placed inside embryos incubator. Embryos monitoring was set to take picture every 20 minutes. Fertilization as well as pronuclear morphology was assessed 16-19 hours after ICSI. The next morphological assessment was conducted on second day (44-48 hours post ICSI) or third day (64-72 hours post ICSI).

For morphokinetic monitoring using time-lapse technique, pictures sequences were recorded digitally and were analyzed by embryologists or competent doctors. We monitored time of cell cleavage. The first cell division into 2 cell was marked into t2. Second division into 3 cell was marked into $\mathrm{t}_{3}$ and so on, third division (4 cell, $\mathrm{t} 4$ ), and fourth division ( 5 cell, $\mathrm{t} 5$ ).

Duration of second cell cycle (cc2) was defined by the time of division from 2 blastomere embryo into 3 blastomere embryo (cc2 = t3 - t2). Second synchrony (s2) was defined by time of transition phase from 2 blastomere embryo into 4 blastomere embryo (s2 = t4 $\left.-\mathrm{t}_{3}\right)$.

Embryos categorized were based on time-lapse monitoring with $\mathrm{t}_{5}, \mathrm{s2}$, and $\mathrm{cc} 2$ parameters. These parameters were inputted into algorithm based on Meseguer et al. 


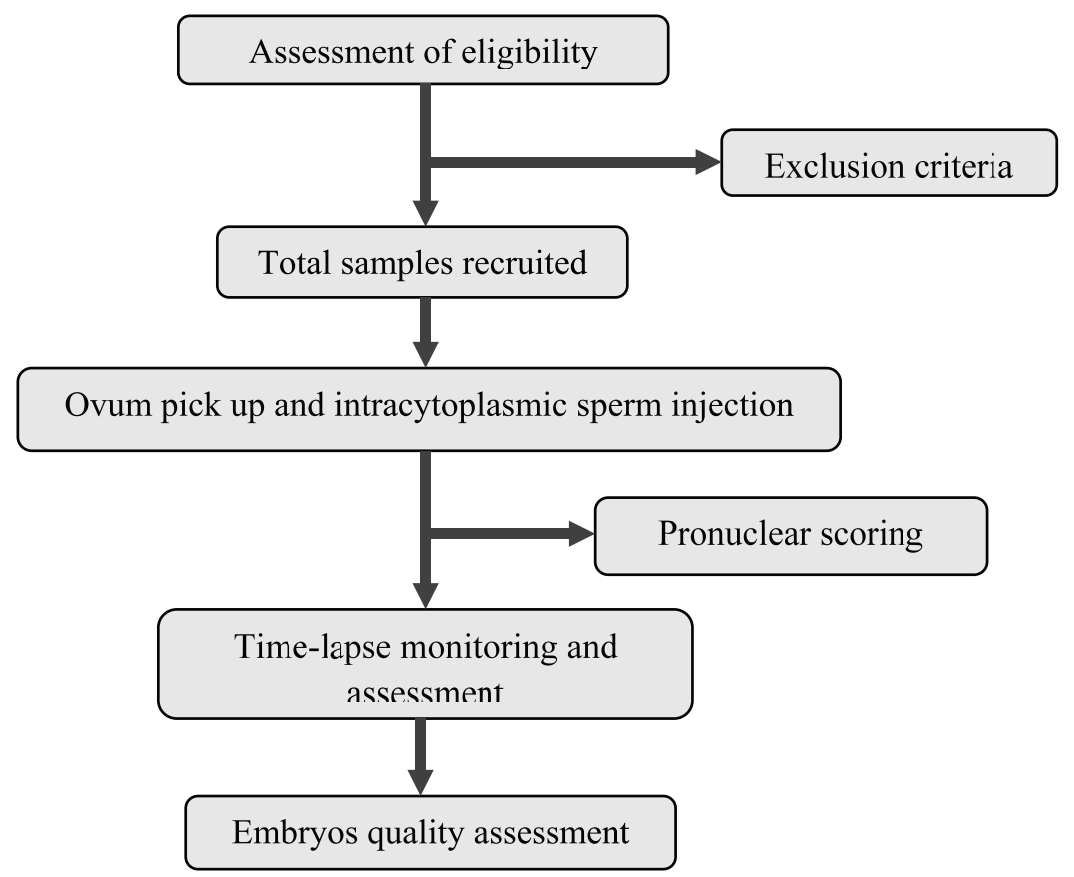

Figure 1: Flow chart of the study.

[10]. The classified embryos then converted into two subgroups, good or bad predictor (categorical data).

For conventional pronuclear morphology assessment, we assessed pronuclear morphology 17-19 hours post ICSI. Pronuclear morphology was classified into 3 scoring based on Alpha Scientist in Reproductive Medicine and ESHRE Special Interest Group Embryology consensus [11]. Score 1 would be converted into good predictor, while score 2 or 3 would be converted into bad predictor (categorical data).

Next, embryos were evaluated in second or third day after ICSI. This evaluation was to identify embryos quality as final evaluation of this study. Embryos quality was assessed using static morphology with Veeck grading [12]. Veeck grading consists of 5 categories, the best category was scored with 1 and the worst was 5 . For the study purpose, category 1 and 2 would be classified into good quality embryos hence 3-5 would be classified into bad quality embryos (categorical data). Schematic view of this study can be seen in Figure 1.

Data collected was analyzed using SPSS software for windows version 22. Data was analyzed with Fisher-exact test (non-parametric) to assess distribution and comparison. To assess the correlation between each variable we used Spearman test.

\section{Results}

In this study, we collected 72 embryos from 19 endometriosis patients whom visited Yasmin IVF Clinic, Cipto Mangunkusumo National General Hospital, Jakarta to get infertility treatment benefited from IVF. Mean age of endometriosis patients was 35 years old. From 72 embryos retrieved we assessed pronuclear scoring and morphokinetics 


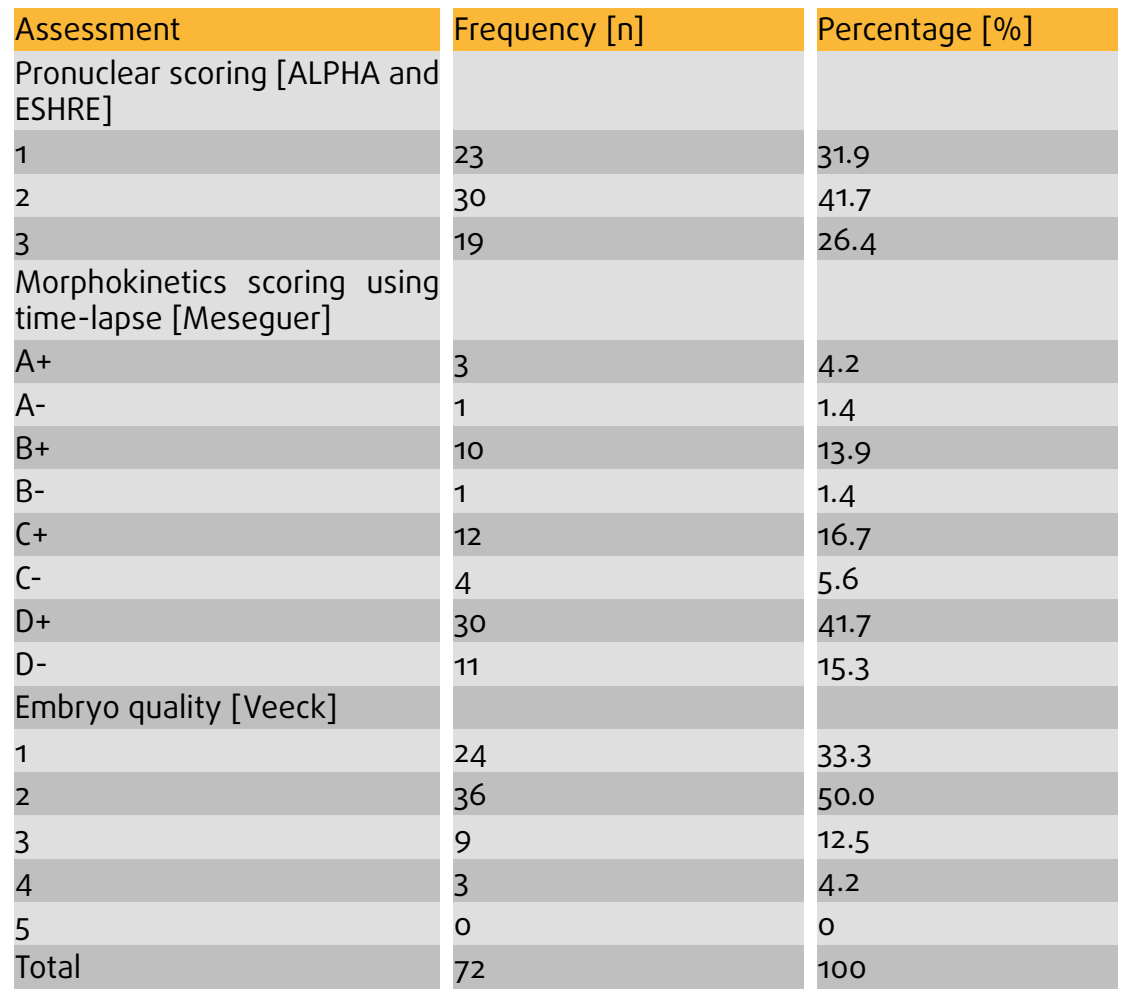

TABLE 1: Distribution of embryo characteristics of endometriosis patients.

Assessment
Pronuclear
Good
Poor
Morphokinetics
Good
Poor

\begin{tabular}{l|l|}
$\begin{array}{l}\text { Embryo Quality } \\
\text { Good }\end{array}$ & Poor \\
\hline 21 & 2 \\
39 & 10 \\
\hline 3 & 1 \\
57 & 11
\end{tabular}

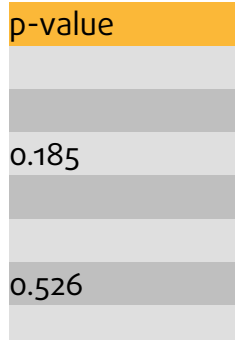

TABLE 2: Comparison of pronuclear scoring and morphokinetics assessment using time-lapse toward embryo quality of endometriosis patients.

using time-lapse. At the end of observation, we assessed static embryos quality using Veeck grading. Table 1 shows the characteristics of the embryos.

From Table 1 we found that most of the embryos had pronuclear scoring of 2, morphokinetics using time-lapse of $\mathrm{D}+$, and embryo quality of 2.

For further analysis we categorized pronuclear scoring, morphokinetics, and embryo quality into good or poor quality. Embryo with pronuclear scoring 1 would be categorized into good quality, hence the rest would be categorized into poor quality. A+ and Aembryos from morphokinetic assessment would be categorized into good quality, the rest ( $\mathrm{B}+$ to $\mathrm{D}-$ ) would be categorized into poor quality. Embryo quality of Veeck grading 1 and 2 were considered good quality, thus the rest (grade $3,4,5$ ) were considered poor quality.

After data were classified, we compared pronuclear scoring and morphokinetic assessment toward embryo quality, as seen on Table 2. 


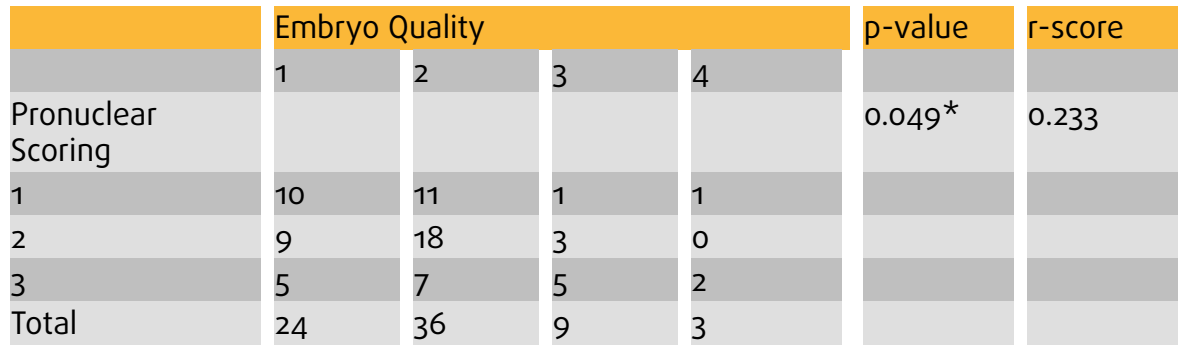

TABLE 3: Correlation of pronuclear scoring with embryo quality of endometriosis patients.

\begin{tabular}{|c|c|c|c|c|c|c|}
\hline & \multicolumn{4}{|c|}{ Embryo Quality } & \multirow[t]{2}{*}{ p-value } & \multirow[t]{2}{*}{ r-score } \\
\hline & 1 & 2 & 3 & 4 & & \\
\hline $\begin{array}{l}\text { Morphokinetics } \\
\text { Assessment }\end{array}$ & & & & & 0.563 & 0.069 \\
\hline$A+$ & 1 & 1 & 0 & 1 & & \\
\hline$A^{-}$ & 0 & 1 & 0 & 0 & & \\
\hline$B+$ & 6 & 3 & 1 & 0 & & \\
\hline B- & 1 & 0 & 0 & 0 & & \\
\hline C+ & 2 & 10 & 0 & 0 & & \\
\hline C- & 1 & 1 & 2 & 0 & & \\
\hline$D+$ & 9 & 14 & 6 & 1 & & \\
\hline D- & 4 & 6 & 0 & 1 & & \\
\hline Total & 24 & 36 & 9 & 3 & & \\
\hline
\end{tabular}

TABLE 4: Correlation of morphokinetics assessment with embryo quality of endometriosis patients.

Based on Table 2, we conducted bivariate analysis to compare each assessment toward embryo quality. There were some cells with expected count less than 5 so we used Fisher-exact test to analyze. We found that every single assessments (pronuclear or morphokinetics) had p-value of more than 0.05 . So, there were no significant difference between pronuclear and morphokinetics assessment toward embryo quality of endometriosis patients.

We conducted another analysis without categorizing pronuclear scoring and morphokinetics assessment to correlate with embryo analysis. We used Spearman test to analyze the correlation and we found that pronuclear scoring had significant correlation with embryo quality with p-value of 0.049 and r-score 0.233 . This result showed that pronuclear scoring has weak correlation with embryo quality of endometriosis patients. Details of the data can be seen on Table 3.

We also correlate morphokinetics assessment using time-lapse monitoring with embryo quality. From Spearman test, we found that there was no correlation between morphokinetics assessment and embryo quality with p-value of 0.22 . Details of the data can be seen on Table 4 .

From the analysis above we found that neither pronuclear scoring nor morphokinetics assessment using time-lapse monitoring showed a good predictor for embryo quality of endometriosis patients. However, there was a weak correlation between pronuclear scoring and embryo quality of endometriosis patients. 


\section{Discssions}

The aim of this study was to compare conventional pronuclear assessment and morphokinetics assessment using time-lapse monitoring toward embryos quality of endometriosis patients whom underwent in vitro fertilization. Parameters assessed were pronuclear scoring based on ALPHA and ESHRE consensus [11], morphokinetics assessment using time-lapse monitoring based on optimal cleavage time from Meseguer et al. [10], and embryo quality based on Veeck grading [12].

From this study, we found that there was a weak correlation between pronuclear scoring and embryo quality of endometriosis patients $(p=0.049)$. This result was similar to study conducted by Tesarik, Balaban, and Khalili. They stated that normality of embryos during pronuclear stage were associated with further embryos development [13-15]. Systematic review from Nicoli et al. also supported our result. There were 15 from 25 studies (60\%) showed that there was significant correlation between pronuclear and embryo quality and cleavage. 15 from 20 studies (75\%) also showed its correlation to blastocyst formation and quality. [16]

PN scoring of 3 were considered abnormal due to lack of nucleolar precursor body (NPB) or only have one NPB. Those embryos show inability to cleave on second or third day. This result was similar to Svarcova et al., zygotes that had no NPB or one NPB associated with epigenetic defect and abnormal embryo development in animal model. [17]

Morphokinetics parameter using time-lapse monitoring may predict genetically normal and viable embryos through cellular processes showed from cell cycles. [10] Research conducted by Montag, who observed embryos development simultaneously and compare with static morphology, showed different embryos morphologies on 2PN stage (25-33\%) and two-cells stage (42.4\%). From this study can be inferred that static embryo has limitation because every single embryo has different time to develop that cannot be generalized. Hence, embryos monitoring using time-lapse were considered the most objective assessment to choose good quality embryos [18].

Other studies also showed that $t_{2}$ and t3 parameters on morphokinetics assessment were used to evaluate embryos development. Those studies showed statistically significant results toward embryos development, implantation, or both. [10, 19-25] However, there was two studies that contradict the results of the other studies due to lack of sample $[26,27]$.

Our results showed that there were no significant correlation between morphokinetics assessment using time-lapse and embryos quality of endometriosis patients ( $p>0.05)$. This might be resulted due to limited number of samples that can be corrected by adding more samples and lengthening the duration of samples collection. Another factor that might contribute is the endometriosis itself leads to poorer oocytes and embryos quality.

Folliculogenesis in endometriosis patients were considered defected that may imply to poor quality oocytes. This is shown by lower granulosa cell percentage on G2/M phases compared to other infertile conditions without ovarian lesion. Low percentage of granulosa cell indicates abnormalities in molecules which regulate G2/M phases i.e. 
cyclin B, cdc2, cdc2s, Weel kinase, and MOS [28]. In endometriosis patients, there is defect on signaling process to DNA synthesis, thus the cells are deviated from normal cell cycle between $S$ and $\mathrm{G}_{2} / \mathrm{M}$ phases, followed by cell apoptosis. Cell with $\mathrm{S}$ phase shortening is more prone to incomplete replication resulting DNA abnormality. This cell is incompetent to advance into blastocyst stage. In addition, it also delays $\mathrm{G} 2$ and $M$ phases [29].

Another weakness of this study is the probability of bias during observation. Our time-lapse monitoring machines only capture image every 20 minutes. So, there was a possibility of some phases that weren't captured by camera. This factor should be considered on the next study.

\section{Conclusion}

Based on those two assessments, neither pronuclear scoring nor morphokinetics assessment using time-lapse monitoring could predict embryo quality of endometriosis patients whom underwent in vitro fertilization. However, there was a weak correlation between pronuclear scoring and embryo quality of endometriosis patients. Morphokinetics assessment using time-lapse monitoring showed no correlation with embryo quality of endometriosis patients.

From our results, pronuclear scoring may be used to predict embryos quality of endometriosis patients in spite of its weak correlation. Considering the limitations, conventional pronuclear scoring is still applicable compared to time-lapse monitoring in endometriosis patients.

\section{Acknowledgment}

The authors thank to Eliza Mansyur and Tita Yuningsih from Yasmin IVF Clinic, Cipto Mangunkusumo National General Hospital for their invaluable expert technical assistance.

\section{References}

[1] J. A. W. Stilley, J. A. Birt, and K. L. Sharpe-Timms, Cellular and molecular basis for endometriosis-associated infertility, Cell and Tissue Research, 349, no. 3, 849-862, (2012).

[2] R. A. Lobo, Endometriosis: etiology, pathology, diagnosis, management, in Comprehensive Gynecology, Mosby, Philadelphia, PA, 2007, 5th ed, chap 19.

[3] A. W. Nap, P. G. Groothuis, A. Y. Demir, J. L. H. Evers, and G. A. J. Dunselman, Pathogenesis of endometriosis, Best Practice and Research: Clinical Obstetrics and Gynaecology, 18, no. 2, 233-244, (2004).

[4] S. Gupta, J. M. Goldberg, N. Aziz, E. Goldberg, N. Krajcir, and A. Agarwal, Pathogenic mechanisms in endometriosis-associated infertility, Fertility and Sterility, 90, no. 2, 247-257, (2008). 
[5] H. M. Harb, I. D. Gallos, J. Chu, M. Harb, and A. Coomarasamy, The effect of endometriosis on in vitro fertilisation outcome: A systematic review and metaanalysis, BJOG: An International Journal of Obstetrics and Gynaecology, 120, no. 11, 1308-1320, (2013).

[6] X. Dong, X. Liao, R. Wang, and H. Zhang, The impact of endometriosis on IVF/ICSI outcomes, International Journal of Clinical and Experimental Pathology, 6, no. 9, 19111918, (2013).

[7] A. Ajduk and M. Zernicka-Goetz, Quality control of embryo development, Molecular Aspects of Medicine, 34, no. 5, 903-918, (2013).

[8] C. Pribenszky, E. Losonczi, M. Molnár, Z. Lang, S. Mátyás, K. Rajczy, K. Molnár, P. Kovács, P. Nagy, J. Conceicao, and G. Vajta, Prediction of in-vitro developmental competence of early cleavage-stage mouse embryos with compact time-lapse equipment, Reproductive BioMedicine Online, 20, no. 3, 371-379, (2010).

[9] J. G. Lemmen, I. Agerholm, and S. Ziebe, Kinetic markers of human embryo quality using time-lapse recordings of IVF/ICSI-fertilized oocytes, Reproductive BioMedicine Online, 17, no. 3, 385-391, (2008).

[10] M. Meseguer, J. Herrero, A. Tejera, K. M. Hilligsøe, N. B. Ramsing, and J. Remoh, The use of morphokinetics as a predictor of embryo implantation, Human Reproduction, 26, no. 10, 2658-2671, (2011).

[11] Alpha Scientist in Reproductive Medicine and ESHRE Special interest Group Embryology. Istanbul consensus workshop on embryo assessment: proceedings of an expert meeting. Reproductive Biomedicine Online, 22, 632-646

[12] L. L. Veeck, Preembrio grading and degree of cytoplasmic fragmentation, In: An Atlas of Human Gametes and Conceptuses: An Illustrated Reference for Assisted Reproductive Technology, Parthenon Publishing, New York, USA, 1999.

[13] J. Tesarik and E. Greco, The probability of abnormal preimplantation development can be predicted by a single static observation on pronuclear stage morphology, Human Reproduction, 14, no. 5, 1318-1323, (1999).

[14] B. Balaban, B. Urman, A. Isiklar, C. Alatas, S. Aksoy, R. Mercan, A. Mumcu, and A. Nuhoglu, The effect of pronuclear morphology on embryo quality parameters and blastocyst transfer outcome, Human Reproduction, 16, no. 11, 2357-2361, (2001).

[15] M. A. Khalili, F. Mardanian, V. Razavi, and N. Esfandiari, The predictive value of pronuclear morphology screening on embryo development and pregnancy outcome in ART cycles, Middle East Fertility Society Journal, 13, no. 1, 44-51, (2008).

[16] A. Nicoli, S. Palomba, F. Capodanno, M. Fini, A. Falbo, and G. B. La Sala, Pronuclear morphology evaluation for fresh in vitro fertilization (IVF) and intracytoplasmic sperm injection (ICSI) cycles: A systematic review, Journal of Ovarian Research, 6, no. 1, article no. 64, (2013).

[17] O. Svarcova, A. Dinnyes, Z. Polgar, S. Bodo, M. Adorjan, Q. Meng, and P. MaddoxHyttel, Nucleolar re-activation is delayed in mouse embryos cloned from two different cell lines, Molecular Reproduction and Development, 76, no. 2, 132-141, (2009).

[18] M. Montag, J. Liebenthron, and M. Köster, Which morphological scoring system is relevant in human embryo development? Placento, 32, no. 3, S252-S256, (2011). 
[19] S. L. Chavez, K. E. Loewke, J. Han, F. Moussavi, P. Colls, S. Munne, B. Behr, and R. A. Reijo Pera, Dynamic blastomere behaviour reflects human embryo ploidy by the four-cell stage, Nature Communications, 3, article no. 1251, (2012).

[20] M. Cruz, N. Garrido, J. Herrero, I. Pérez-Cano, M. Muñoz, and M. Meseguer, Timing of cell division in human cleavage-stage embryos is linked with blastocyst formation and quality, Reproductive BioMedicine Online, 25, no. 4, 371-381, (2012).

[21] M. Dal Canto, G. Coticchio, M. Mignini Renzini, E. De Ponti, P. V. Novara, F. Brambillasca, R. Comi, and R. Fadini, Cleavage kinetics analysis of human embryos predicts development to blastocyst and implantation, Reproductive BioMedicine Online, 25, no. 5, 474-480, (2012).

[22] D. Hlinka, B. Kal'atová, I. Uhrinová, S. Dolinská, J. Rutarová, J. Řezáčová, S. Lazarovská, and $M$. Dudáš, Time-lapse cleavage rating predicts human embryo viability, Physiological Research, 61, no. 5, 513-525, (2012).

[23] K. Kirkegaard, U. S. Kesmodel, J. J. Hindkjær, and H. J. Ingerslev, Time-lapse parameters as predictors of blastocyst development and pregnancy outcome in embryos from good prognosis patients: A prospective cohort study, Human Reproduction, 28, no. 10, 2643-2651, (2013).

[24] I. Rubio, R. Kuhlmann, I. Agerholm, J. Kirk, J. Herrero, M.-J. Escribá, J. Bellver, and M. Meseguer, Limited implantation success of direct-cleaved human zygotes: A timelapse study, Fertility and Sterility, 98, no. 6, 1458-1463, (2012).

[25] C. C. Wong, K. E. Loewke, N. L. Bossert, B. Behr, C. J. De Jonge, T. M. Baer, and R. A. R. Pera, Non-invasive imaging of human embryos before embryonic genome activation predicts development to the blastocyst stage, Nature Biotechnology, 28, no. 10, 1115-1121, (2010).

[26] A. Campbell, S. Fishel, N. Bowman, S. Duffy, M. Sedler, and S. Thornton, Retrospective analysis of outcomes after IVF using an aneuploidy risk model derived from time-lapse imaging without PGS, Reproductive BioMedicine Online, 27, no. 2, 140-146, (2013).

[27] S. Chamayou, P. Patrizio, G. Storaci, V. Tomaselli, C. Alecci, C. Ragolia, C. Crescenzo, and A. Guglielmino, The use of morphokinetic parameters to select all embryos with full capacity to implant, Journal of Assisted Reproduction and Genetics, 30, no. 5, 703-710, (2013).

[28] M. Toya, H. Saito, N. Ohta, T. Saito, T. Kaneko, and M. Hiroi, Moderate and severe endometriosis is associated with alterations in the cell cycle of granulosa cells in patients undergoing in vitro fertilization and embryo transfer, Fertility and Sterility, 73, no. 2, 344-350, (2000).

[29] N. M. Basile, Time-lapse technology: evaluation of embrio quality and new markers for embrio selection, Expert Rev Obstet Gynecol, 7, no. 2, 175-90, (2012). 